\title{
LA REACCIÓN QUÍMICA: DESCRIPCIONES E INTERPRETACIONES DE LOS ALUMNOS DE LICEO
}

\author{
CHASTRETTE, M. y FRANCO, $M$. \\ Universidad Claude Bernard LYYN I. LIRDIS. 43 Bd. du 11 Novembre 1918. \\ 69622 Villeurbanne, France.
}

\section{SUMMARY}

We have carried out a piece of research about the ass milation of the chemical reaction by secondary students, based on the study of their spontaneous descriptions of the process, their ability to cxplain it in corpuscular terms and of correctly applying the law of matter preservation

The experimental sample were 19 students between 13 and 14 years of age who had been introduced to the subject the previous year. We have introduced a variant in the technique of interviews.

\section{INTRODUCCIÓN}

Los estudiantes de todos los niveles de enseñanza entcuentran dificultades para apropiarse del modelo corpuscular de la materia y del concepto de átomo.

Un estudio sobre las representaciones de los alumnos acerca del átomo, que realizamos en Montevideo (Uruguay) en 1989 , nos ha mostrado que estas dificultades aparecen desde que las nociones mencionadas son abordadas en los cursos de introducción a las ciencias físicas. 1.as representaciones que construyen los jóvenes alumnos se convierten así en obstáculos difíciles de sortear a través de una enseñanza tradicional.

En Ios últimos años han sido llevadas a cabo varias investigaciones didácticas acerca de las representaciones sobre los fenómenos físicos y sus interpretaciones en relación con la naturaleza corpuscular de la materia. Pero hasta este momento, muy pocos trabajos han tenido como objetivo conocer las explicaciones y las interpre. taciones corpusculares que los alumnos proveen acerca de las reacciones químicas.

A través de un análisis bibliográfico profundo de las investigaciones realizadas anteriormente acerca de la representaciones del concepto de átomo, hemos encontrado que los alumnos de liceo saben pocas cosas al respecto. Muy pocos de ellos admiten la existencia de espacio vacio entre los átomos (Franco 1990, Barboux 1987, Novick y Nussbaum 1981). Muchos mencionan los constituyentes subatómicos, pero demuestran una gran confusión en lo que respecta a la estructura atómica (Franco 1990). El modelo de Bohr predomina aún entre los estudiantes universitarios, después de haber discutido el modelo de Schroedinger durante los cursos (Cros et al. 1986 y 1988). Algunos autores atribuyen la fuerte persistencia de este modelo a la relativa facilidad dc recordarlo y a la gran frecuencia con que los medios de comunicación y los textos utilizan este tipo de imagen (Cros et al. 1988, Lahore 1988).

En to que respecta a las representaciones sobre la estructura corpuscular de la materia, varios autores han encontrado que los alumnos no establecen una distinción entre las propiedades de las sustancias y las propiedades de las partículas (De Vos y Verdonk 1987, Gabel 1989, Novick y Nussbaum 1979,1981$)$. Un átomo posece las mismas propiedades (cstado físico, color, maleabilidad, etc.) que la sustancia que constituye. Essta concepción que mezcla el sistema de referencia factual (macroscópico) con el referente atomista (microscópico) ha sido denominada «sustancialismo» (Franco 1990, Furió et al. 1989). Aun cuando el término átomo es utilizado frecuentemente en los cursos de química, los adolescentes no han abandonado totalmente el modelo continuo de la 
materia. Fn esta perspectiva el conflicto que surge de la existencia simultánca de un modelo eintuitivo-continuo» y otro «científico-discontinuo» es resuelto por el alumno considerando las partículas como pequeñas por* ciones de la sustancia.

Los trabajos sobre el concepto de reacción química muestran que muchos estudiantes poseen grandes dificultades para comprender sus aspectos fundamentales, aún al finalizar Ios estudios de secundaria (Ben-Zvi 1986, Gabel 1987, Meheut 1989).

El análisis bibliográfico y los resultados de nuestra investigación nos condujeron a continuar nuestro trabajo a través de una serie de experiencias sobre diversas reacciones químicas con Ia finalidad de averiguar:

--cuáles son las descripciones espontáneas de los alum* nos frente a la reacciones químicas,

-en qué medida ellos son capaces de explicarlas en términos corpusculares,

-hasta qué punto poseen la idea de conservación de los elementos en estas transformaciones.

\section{MATERIALES Y MÉTODOS}

Las experiencias fueron realizadas en Lyon, con 19 alumnos del tercer año de secundaria (13-14 años). Es importante mencionar que en el curso anterior de ciencias físico-químicas las nociones de átomo y molécula habían sido introducidas.

Como procedimiento de recolección de datos hemos creado una variante de la técnica de entrevistas ante fenómeno. Para cada una de las cuatro sesiones propusimos una secuencia de observación-descripción-explicacion de las experiencias elegidas. El trabajo experimental fue acompañado de una serie de preguntas de respuesta abierta corta. Un estudio rápido de las respuestas de los alumnos después de la primera sesión nos lievó a entrevistar a algunos de ellos con la finalidad de profundizar el análisis de las respuestas.

Las situaciones experimentales elegidas fueron:

1. Reacción entre nitrato de plomo y yoduro de potasio (De Vos y Verdonk 1985).

1.a. Reacción entre los prođuctos sólidos.

1.b. Reacción entre los sólidos en medio acuoso.

En la primera parte pedimos a los alumnos que anotaran sus observaciones y que imaginaran qué podría haber en un grano de cada uno de los productos sólidos que participan de la reacción para favorecer la movilización de las nociones corpusculares. Fstas experiencias son utilizadas por De Vos y Verdonk (1985) como parte de una estrategia de enseñanza, con muy buenos resultados.

\section{Ciclo del cobre.}

2.a. Reacción del cobre con ácido nítrico.

2.b. Electrólisis de la solución de nitrato de cobre obtenida.

El objetivo de esta experiencia ha sido estudiar las representaciones de los alumnos cuando la conservación de un elemento es puesta en evidencia a través de uná serie de reacciones químicas.

3. El cinc y el yodo.

3.a. Reacción de formación de yoduro de cinc a partir de los cuerpos simples que lo constituyen.

3.b. Flectrólisis de la solución de yoduro de cinc obtenida.

Antes de llevar a cabo la parte b, hemos pedido a los alumnos que hicieran predicciones acerca de lo que ocuriría.

4. Cloruro de calcio y bicarbonato de sodio.

4.a. Reacción entre los productos sólidos al ponerlos en contacto con una solución acuosa de rojo de fenol.

4.b. Reconocimiento de los componentes de reactivos y productos (ensayos a la llama, reacción con ácido clorhídrico).

Antualmente un gran número de alumnos de liceo realiza la experiencia 4.a. propuesta por Barber (1986) en Lawrence Hall of Science en Berkeley, obteniéndose buenos resultados.

Por medio de estas experiencias buscamos averiguar en qué medida los alumnos pueden reconocer en una reacción química la transformación de las sustancias y la conservación de los elementos.

El análisis a priori de las situaciones experimentales elegidas nos llev6 a enunciar un conjunto de hipótesis que buscamos validar a través de nuestra investigación:

- Ias transformaciones físicas y la reacciones químicas no son bien diferenciadas por los alumnos,

-las explicaciones corpusculares no son espontáneas,

-las explicaciones corpusculares que se pueden inducir son fundamentalmente sustancialistas.

\section{RESULTADOS}

1. Reacción entre nitrato de plomo y yoduro de potasio.

Los resultados fueron analizados de acuerdo con la categorización propuesta por Andersson (1986). 
L.a mayoría de los alumnos ha descrito la reacción en términos de modificación de sustancias. El 50\% de ellos retomó la descripción macroscópica que habia anotado antes, frecuentemente en forma de dibujo. Muchas explicaciones atribuyen a las partículas tas características macroscópicas de las sustancias, lo que ya hemos mencionado como «sustancialismo». Solamente dos alumnos han suministrado explicaciones que tienen en cuenta la interacción entre las partículas. Es importante destacar que entre los alumnos $(51 \%)$ que habían imaginado la existencia de partículas en los sólidos reaczionantes, el $80 \%$ no ha utilizado ninguna explicación corpuscular de la transformación observada.

I as descripciones de los alumnos sobre la experiencia $b$ incluyen a menudo las nociones de disolución y difusión (37\%), pero estos fenómenos no son explicados cliaramente. Hemos encontrado también una confusión entre disolución y fusión en $22 \%$ de los casos. La mayor parte de los alumnos explica la interacción entre los reactivos como una simple mezcla. Ningún alumno ha propuesto una interpretación corpuscular de esta transformación, aún aquéllos que habian suministrado este tipo de explicación para la primera experiencia.

Durante las entrevistas que siguieron a esta primera sesion hemos empleado "contrasugerencias» (Dolle y Bellano 1989), como si hubieran sido suministradas por un miembro de la clase, para enfrontar al adolescente a una explicación en términos corpusculares. Aun aquéllos que accptaron este punto de vista para explicar lo ocurrido en la experiencia anterior no han sido capaces de explicar la segunda situación haciendo uso del modelo introducido.

\section{Ciclo del cobre.}

La primera parte de csta experiencia provee varios signos que permiten reconocer una reacción quírnica. $l$ as descripciones de los cambios macroscópicos ocurridos han sido en su totalidad adecuados. Pero, en cambio, solamente un alumno reconoció la reacción química que tuvo lugar como tal.

La aparición de una coloración rojiza o marrón sobre el electrodo de grafito durante la electrólisis đe la solución resultante fue anotada por el $80 \%$ de los alumnos. El $69 \%$ de las explicaciones señalan la presencia del cobre en la solución y sobre el grafíto. El $57 \%$ de las explica. ciones atribuye la causa del depósito de cobre a la electricidad.

\section{El cinc y el yodo.}

Las descripciones de las sustancias originales y de los cambios ocurridos durante la reacción entre ellas fueron todas adecuadas. Durante la điscusión previa a la electrólisis, varios alumnos adelantaron que recuperaríamos el cinc, por analogía con Ia experiencia 2. Después, hemos encontrado que la mitad de los alumnos mencionó directamente la reaparición del cinc, pero solamente un $17 \%$ explicó este acontecimiento considerando la presencia de dicho elemento en la solución.

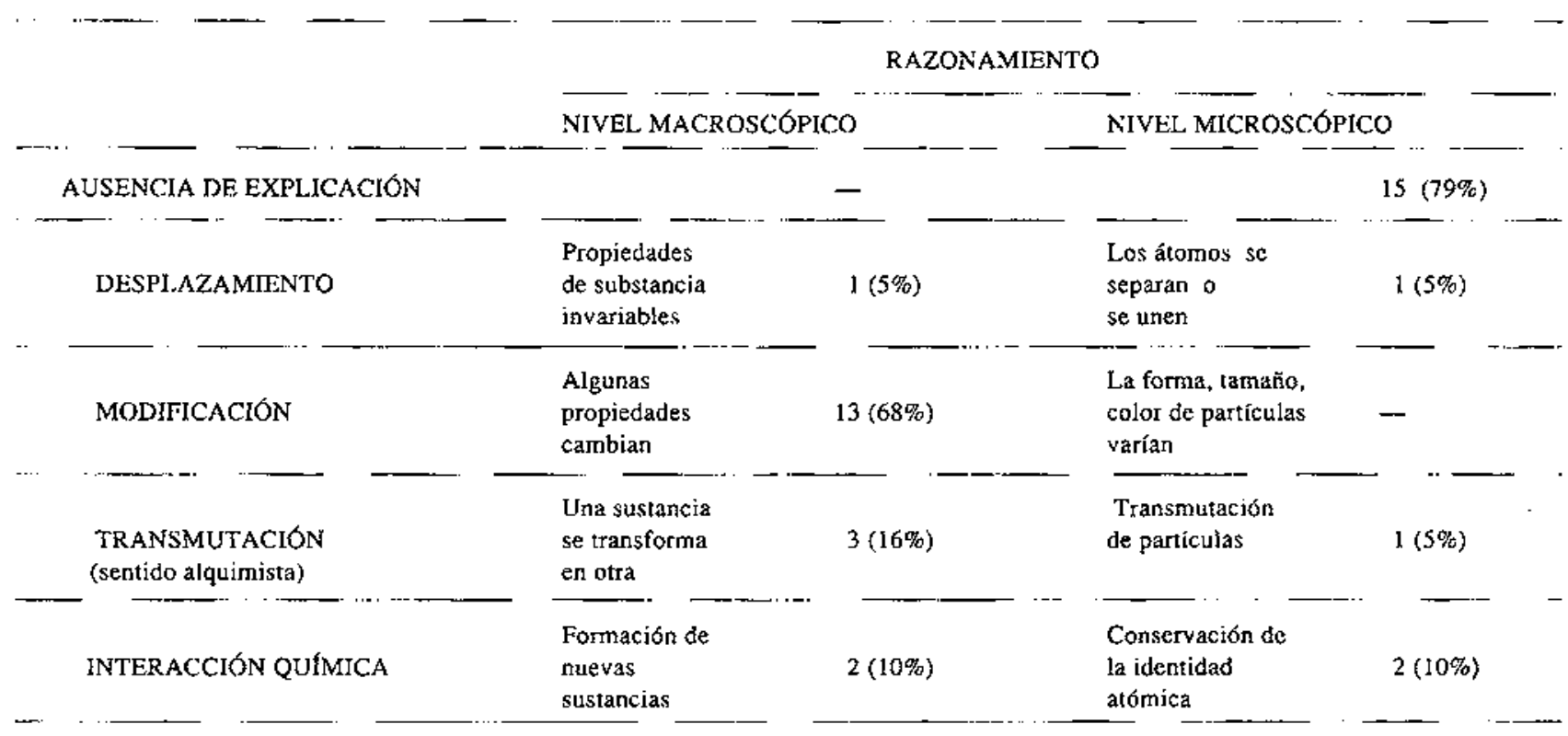


La reaparición del yodo fue menos evidente $(28 \%)$. Varios alumnos no propusieron ninguna explicación y otros reformularon sus descripciones en lugar de hacerto. Tres jóvenes mencionaron específicamente la recuperación de las sustancias de partida.

\section{Cloruro de calcio y bicarbonato de sodio.}

La totalidad de los alumnos ha descrito de manera apropiada los signos que se observan durante la reacción. Todas las explicaciones corresponden a un razona* miento a nivel macroscópico, sin que ningún alumno haya propucsto una explicación corpuscular. Siete jóvenes explicaron el fenómeno observado como una interacción química mientras que otros seis retomaron sus descripciones macroscópicas, $o$ bien no proveyeron ninguna explicación (Cuadro II).

\section{Cuadro II}

Resultados comparativos de lats explicaciones de los alumnos sobre las sesiones 1 y 4 .

RA\%ONAMIINTO A NIVEL MACROSCÓPICO

Nitrato de plomo- Cloruro de calcio-

yoduro de potasio bicarbonato de sodio

(1" sesion)

$\left(2^{n}\right.$ sesion)

\begin{tabular}{|c|c|c|}
\hline $\begin{array}{l}\text { ALSYINCIA DE } \\
\text { EXPLICACION }\end{array}$ & - & $6(32 \%)$ \\
\hline DESPLAZAMIFNTO & $1(5 \%)$ & - \\
\hline MODDIFICACION & $13(68 \%)$ & $5(26 \%)$ \\
\hline TRANSMUTACION & $3(16 \%)$ & $1(5 \%)$ \\
\hline INTIERACCION QUÍMICA & $2(10 \%)$ & $7(37 \%)$ \\
\hline
\end{tabular}

\section{DISCUSIÓN}

Los resultados de la investigación muestran que el método utilizado es adecuado para detectar las representaciones de los alumnos sobre las reacciones químicas. Se trata de un método bien aceptado por los adolescentes. El hecho de trabajar en una situación que se asemeja a una situación de clase hace que las respuestas sean espontáneas y los efectos de perturbación que caracterizan las entrevistas con alumnos de esta edad no se manifiesten. Además, Ia observación de los alumnos en pares junto con los datos recogidos a través de las respuestas individuales a los cuestionarios propuestos permiten conocer en qué grado el alumno está convencido de sus propias interpretaciones.

La secuencia de experiencias elegida permite apreciar diversos aspectos de las representaciones de los alumnos. Las dos primeras experiencias sirven para estudiar la manera en que los alumnos describen e interpretan las reacciones químicas. La tercera actividad pone en jucgo, no sólo los aspectos descriptivo e interpretativo, sino también la capacidad del alumno de hacer predicciones. Finalmente, la última experiencia permite evaluar si los dos aspectos de una reacción química, el de transformación de las sustancias y el de conservación de los elementos, forman parte de las representaciones de los alumnos.

Fin todos los casos hemos encontrado que las descripciones de los alumnos sobre las transformaciones que observan, incluyen las interpretaciones que ellos elaboran accrca de la situación. Desde un punto de vista cpistemológico, esto significa que para los alumnos, el nivel de los hechos (referente factual) no se diforencia del nivel microscópico (referente atomista). Los conocimicntos teóricos adquiridos anteriormente influyen fuertemente sobre sus observaciones.

Las descripciones en sí mismas son generalmente adecuadas. Los estudiantes describen mejor las experiencias en que ocurren pocos cambios observables que aquéllas donde varios signos externos aparecen simultáneamente. Tn este último caso, un aspecto de la reacción atrac la atención del joven más que los otros y en consecuencia sus observaciones se dirigen haciá él, como fue observado por Kempa y Ward (1988).

I.os alumnos no explican las transformaciones que observan haciendo uso de un modelo de partículas, aunque hayan abordado su estudio con anterioriđad. Solamente provecn este tipo de interpretación cuando son inducidos a hacerlo, y en este caso sus explicaciones son sustancialistas. $\mathrm{l}$ a idea de conservación de las sustancias durante una reacción química es bastante fuerte. A lo largo de la investigación, hemos encontrado una sensible evolución de esta noción. l a presentación de una reacción en la cual se aprecian a la vez, la transformación de las sustancias y la conservación de loseiementos polariza las explicaciones de los alumnos: o bien reconocen que ha habido formación de nuevas sustancias, o de lo contrario, no son capaces de elaborar ninguna explicación (Cuadro II).

\section{CONCLUSIÓN}

Por medio del análisis de los resultados que acabamos de discutir podemos concluir que nuestras hipótesis de investigación han sido validadas.

* Los alumnos poseen una gran confusión entre las reacciones químicas y las transformaciones físicas.

* Las observaciones que ellos realizan incluyen a la ve\% sus interpretaciones de los hechos observados.

* En cuanto a las explicaciones microscópicas de las reacciones químicas, los alumnos:

-No las utilizan si no son inducidos a hacerlo y las abandonan espontáneamente una vez que cesa el estimulo inductor. 
-Recurren al sustancialismo para rendir cuenta de las propiedades de las sustancias y de las transformaciones que cllas sufren.

Creemos que es posible desarrollar una estrategia de enseñanza que tome en cuenta estos obstáculos, jara lograr la apropiación de los conceptos de reacción química y de elemento por parte de los alumnos que se inician en el estudio de la química. Si se planifican situaciones de clase que incluyan experiencias, discusiones y debates, así como actividades de modelización

\section{REFERENCIAS BIBIIOGRÁFICAS}

ANDERSSON, B.,1986. Pupils' explanations of some aspects of chemical reactions, Science Education, 70(5), pp. 549-563.

BARBER, I., 1986. Chemical Reaction: Teachers' Guide. L.H.S. GEMS, Lawrence Hall of Science, University of California.

BARBOUX, M., CHOMAT, M., LARCHER, C., MEHELT, M.,1987. Modèle particulaire et activités de modélisation en classe de 4 ème. $I N R P$-DPI - LIRESPT UA 663.

BEN-ZVI, R., BAT-SHEVA, E. y SILBERSTEIN, J., 1986. Is an atom of copper malleable? Journal of Chemical Education, 63, pp. 64-66.

CROS, D., MAURIN, M., AMOUROUX, R., CHASTRETTE, M., LEBER, J. y FAYOL, M., 1986. Conceptions of first year university students of the constituents of matter and the notions of acids and bases, International Journal of Science Education, 8, pp. 305-313.

CROS, D., CHASTRETTE, M. y HAYOL, M.,1988. Conceptions of second year university students of some fundamental notions in chemistry, International Journal of Science Education, 10, pp. 331-336.

DE VOS, W. y VERDONK, A., 1985. A new road to reactions. Part 1, Journal of Chemical Education, 62(3), pp. 238-240).

DE VOS, W. y VERDONK, A., 1987. A new road to reactions. Part 4. The substance and its molecules, Journal of Chemical Education, 64(8), pp. 692-694.

DOLLE, J. M. y BELLANO, D.,1989. Ces enfants qui n'apprennent pas. (Paidós-Centurion; Patis). adecuadas, podrá conseguirse la construcción de estos conceptos fundamentales.

Este trabajo constituye solamente el comienzo de nuestra investigación didáctica. Diversas situaciones didácticas deberán ser propuestas para articular una estrategia de enseñanza cohcrente que logre la construcción progresiva de los conceptos deseados. Esta estrategia deberá ser el objeto de una investigación profunda tendiente a precisar sus términos, a encontrar sus límites y a validarla.
FRANCO, M.,1990. Apprentissage de quelques concepts fondamentaux en chimie: étude des représentations des élèves. Mémoire DEA. Univ. Lyon I.

FURIÓ, C., AZCONA, R., GUISASOLA, G. y MIJIKA, E.,1989. Concepciones de los estudiantes sobre una magnitud no introducida en Química: la cantidad de sustancia, Enseñanza de las Ciencias, extra, tomo 1, pp. 193-194.

GABEL, D.,1987. Let us go back to nature study, Journal of Chemical Education, 64(9), pp. 727-729.

GABEL, D., SAMUEL, K. y HUNN, D.,1989. Understanding the particulate nature of matter. Journal of Chemical Education, 66, pp. $695-697$

KEMPA, R.F. y WARI, J.E., 1988. Observational thresholds in school chemistry, International Journal of Science Education, 10, pp. 275-284

LAHORE, A., 1988. El concepto del átomo en los estudiantes: una encuesta. (Comunicacion personaf).

MEHEUT, M.,1989. Des représentations des élc̀ves au concept de réaction chimique: premières étapes, Bulletin de l'Union des Physiciens, 716, pp. 997-1011.

NOVICK, S. y NUSSBAUM, J.,1979, Toward the diagnosis by science teachers of pupils misconceptions: an exercise with student teachers, International Journal of Science Education, 1, pp. 159-169.

NOVICK, S. y NUSSBAUM, J.,1981. Pupils understanding of the particulate nature of matter: a cross age study, Science Education, 65(2), pp. 187-196. 